\title{
Reducing Utilization by Uninsured Frequent Users of the Emergency Department: Combining Case Management and Drop-in Group Medical Appointments
}

\author{
Steven Crane, MD, Lori Collins, RN, James Hall, PhD, Donald Rochester, MDiv, \\ and Steven Patch, PhD
}

Background: Patients with complex behavioral health and medical problems can have a disproportionate impact on emergency departments.

Methods: We identified a cohort of 255 low-income, uninsured patients who had used inpatient or emergency department services more than 6 times in the previous 12 months. Between July 2010 and June 2011 we enrolled 36 of these high-risk patients to participate in a twice-weekly drop-in group medical appointment staffed by an interdisciplinary team of a family physician, behavioral health professional, and nurse case manager. The team provided 705 patient visits in a group setting (a total of 108 group sessions) and 652 case manager phone calls. The average number of clients per drop-in group medical appointment was 6.5 .

Results: Emergency department use dropped from a rate of 0.58 per patient per month to $0.23(P<$ $.001)$, and hospital charges dropped from $\$ 1167$ per patient per month to $\$ 230(P<.001)$. Employment status increased from 4 to 14 among the 36 patients enrolled. Total annualized cost of the program was $\$ 66,000$.

Conclusions: Team-based drop-in group medical appointments coupled with case management seem to be a cost-effective model to reduce emergency department visits by some patients with complex behavioral health and medical needs. (J Am Board Fam Med 2012;25:184-191.)

Keywords: Chronic Disease, Cost Effectiveness, Emergency Medicine, Health Care Team, Primary Health Care, Quality of Health Care

Patients with complex medical and behavioral health needs can have a disproportionate impact on emergency department (ED) and inpatient services.

This article was externally peer reviewed.

Submitted 1 May 2011; revised 10 November 2011; accepted 16 November 2011.

From the Division of Family Medicine, Mountain Area Health Education Center, Hendersonville, NC (SC); the Margaret R. Pardee Hospital, Hendersonville, NC (SC, LC, $\mathrm{JH}, \mathrm{DR})$; the Department of Mathematics, University of North Carolina, Asheville (SP); and the Department of Family Medicine, University of North Carolina, Chapel Hill (SC).

Funding: This project was funded through grants from the NC HealthNet Program, and the Margaret R. Pardee Hospital Charitable Foundation.

Conflict of interest: none declared.

Corresponding author: Steven Crane, MD, Division of Family Medicine, Mountain Area Health Education Center, 709 N. Justice Street, Suite B, Hendersonville, NC 28791 (E-mail: steve.crane@pardeehospital.org).
Those who are identified as low-income or are uninsured may be more likely to use the ED for nonemergent care because of reduced access to primary care or they have complex social, behavioral health, or physical health needs that are difficult to address in traditional primary care settings.

There is extensive evidence that care management can reduce ED visits in this cohort of patients. ${ }^{1-5}$ Kern Medical Center was able to reduce the risk of ED visits for 98 frequent users over a 1.5 -year period with a care management program. ${ }^{6}$ Coupling housing assistance and care management lead to a $24 \%$ reduction in ED visits in a group of homeless patients in another cohort study. ${ }^{7}$ Care management also seems to be a cost-effective means to improve psychosocial problems that are common among patients with 
frequent ED use. ${ }^{8,9}$ This seems to be true even for patients who are considered refractory to care. $^{10}$

Although care management is an important means to address some of the social and behavioral health issues of these patients, providing medical care for those with both physical and behavioral issues presents additional challenges. Patients may have a variety of barriers that prevent them from accessing traditional primary care venues, particularly those settings that do not allow patients to walk in at their convenience or patients who may need intensive services during a personal crisis. Many patients are unable to afford even a minimal copayment that may be expected at time of a nonemergent outpatient visit and may choose to access the ED where a copayment may not be required. Patients with difficult life circumstances also may be more likely to not show for an appointment.

Group medical visits can be an efficient way to provide medical care and social support for patients with chronic illness. Although some have advocated the use of drop-in group medical appointments (DIGMAs) for patients with undifferentiated medical problems, there seems to be little experience with this model in serving patients with complex behavioral and physical health needs. ${ }^{11}$

We report the first-year results of a coordinated, team-based approach that integrates medical and behavioral health care and case management primarily through drop-in group visits, targeting a cohort of low-income, uninsured patients with previously high rates of ED and inpatient use.

\section{Methods}

\section{Setting}

Margaret R. Pardee Hospital in Hendersonville, North Carolina, is a 200-bed, not-for-profit, countyowned hospital that provided 32,209 ED visits in 2010. The primary catchment area for the hospital is Henderson County, which has a population of approximately 100,000 in an area of 375 square miles. ${ }^{12}$ The hospital sponsors a single, rural-track family residency training program of 9 residents. The nearest hospital is a 90 -bed facility approximately 10 miles away.

\section{Sample}

We identified a total of 255 patients who were uninsured and had used the ED at least 6 times during the 12-month period of July 2009 to June 2010. The median number of visits per person per year for this cohort was 7.6, or 0.63 visits per person per month. The median age was 32 years, and 130 of 255 patients (51\%) were women. Combined ED and inpatient charges for this small group of patients were \$3.19 million (median, $\$ 7513$ per person per year), nearly all of it uncompensated care. This represents $35 \%$ of the $\$ 9$ million in uncompensated care provided by the hospital in 2010, which posted $\$ 128$ million in total operating revenue that year. ${ }^{13}$

\section{Recruitment Methods}

To be eligible for the program, patients needed to belong to this original cohort and have family income of $200 \%$ or below the federal poverty guidelines. We attempted to contact all these patients by phone or by direct contact if they presented to the ED for care or upon discharge from the inpatient unit. We were able to reach only 147 of the original cohort because the remainder did not have a functioning phone number listed in the medical record. Of the 147 contacted, 70 initially expressed interest in the program over the phone, but only 36 completed the enrollment process and attended at least one group visit over the course of the year. Those who agreed to participate in the program received individualized informed consent information from the case manager, provided proof of income to determine eligibility, and were asked to fill out a baseline functional assessment and several behavioral health screening tools, including a 9-item Patient Health Questionnaire, 7-item Generalized Anxiety Disorder measure, and the Mood Disorder Questionnaire. The admission process began in midJune, 2010, with the first DIGMA on July 2, 2010, with enrollment continuing throughout the year.

\section{Intervention Components}

Once patients were enrolled the intervention consisted of 4 components:

1. DIGMA: These visits were scheduled twice a week on Tuesdays and Fridays from noon until 1 PM and were held in a large room that could accommodate up to 20 persons. Patients would present in turn any medical, behavioral, or social issue they wished the group and the care team to address.

2. Direct telephone access to a registered nurse care manager. Patients were given a cell phone number with direct access to the nurse case manager for any 
questions or problems Monday through Friday 8 AM to 5 PM.

3. Small group "life skills and support" sessions with the care manager. These also were scheduled twice a week on Tuesdays and Fridays from 11 AM to noon for clients needing special assistance and support.

4. Short, individual sessions after the group medical visit. Patients who had additional issues that could not be appropriately addressed during the group session could have an individual session with one or more of the care team members including the physician, behavioral health professional, and/or the care manager from 1 PM to 1:30 PM.

Patients could participate in the program even if they already had a primary care provider (PCP); in those cases any care other than emotional or group support that was provided in the program would be reported to or coordinated with the PCP with the patients' consent. All care provided to clients was documented in an electronic medical record, including all phone calls. These records could be accessed by physicians in the ED as needed.

\section{Intervention Team}

The care team included a family physician, a nurse care manager, and 2 behavioral health providers, only one of whom would attend a DIGMA at a time and all whom at had more than 20 years' clinical experience. The behavioral health staff had additional experience in chronic pain management and treating addictions. The program was supported primarily by a 1 -year grant from the North Carolina Health Net program, which funds innovative health care programs directed toward low-income, uninsured populations. Each of the care team members were either self-employed or employed in other positions at the hospital, but they were paid for the time they committed to this program (family physician, 3 hours a week; the registered nurse case manager, 20 hours a week; and the 2 behavioral health professionals, 1.5 hours a week each).

The team generally met before and after each group appointment to review individual patient care plans and discuss the evolving group process/ culture. The behavioral health provider attending the meeting that day would summarize the group process in a secure e-mail to the other behavioral health specialist to maintain continuity from visit to visit. The group appointment included time for each patient to share issues with the group. As time allowed or if the clinical situation called for it, patients at times could also have short individual sessions with one or more members of the care team to discuss medical or behavioral health concerns.

The program was based at the Free Clinics of Henderson County, which was an active partner in the program. They provided office space for the case manager, a meeting room for the DIGMA, and a variety of wrap-around services to enrolled clients, including free prescriptions at the onsite pharmacy and any necessary specialty referrals provided at no charge to patients. The hospital provided laboratory tests and radiology studies at no cost to patients once they applied for the hospital's Charity Care program.

\section{Analysis}

Our primary endpoints for the project were to measure the impact of the program on the rate of ED visits per enrollee per month and total ED and inpatient charges compared with each individual's rate before enrollment. We recorded the number of group visits each patient attended, as well as the number of phone calls made to or by the case manager from or to the client, respectively. Enrolled patients' employment status and secure housing were tracked as functional measures. At the end of the 12-month period we randomly selected 36 patients from the original 255-person cohort who had not been enrolled in the program and measured their ED and inpatient use from July 1, 2010, through June 30, 2011, to serve as a control group.

The 2 variables used to measure success of the program were visits per month and expenses per month. In some cases the expenses were recorded with a time lag of up to 1 month. Therefore, some of the expenses recorded for the intervention group were actually incurred before they began treatment. For example, one of the subjects who had participated for 1 month had no visits and expenses of $\$ 4311$ after treatment. Because of the unreliability of the responses for those who had participated for only a short time, subjects who had participated in the intervention for less than 3 months (2 subjects) were excluded from the statistical analysis. For the other participants, the change in expenses is a conservative measure of the program's impact 
because it is possible that some of those expenses were actually incurred before treatment.

Comparisons of the preintervention variables of sex, age, number of visits per month, and expenses per month were conducted between the group receiving the intervention and the remainder of the cohort. Because of nonnormality in most of the variables, rank sum tests were performed on comparisons for quantitative variables. A 2-sample proportion test was performed to compare ages.

A signed rank test was conducted on the intervention group to compare visits before intervention versus visits after intervention and expenses per month. To compare the changes in the number of visits for those in the intervention group with what might have occurred had they not participated in the intervention, a subgroup of the cohort was selected and the number of visits in the year following the beginning of intervention was tabulated. To be eligible to be in the comparison group the patients had to have some evidence that they were in the area during that year by either having some visits or some contact with the investigators. Thus, it is possible that some of the cohort remained in the area and had no visits or that some were in the area for part of the year but not the whole year. To compare the number of visits before intervention, visits after intervention, and difference in visits between the intervention group and the cohort subset, a rank sum test was performed. Rank sum tests also were performed for age differences and differences in expenses before the intervention to investigate if the same relationships held for the subset as for the full cohort. The treatment program, screening tools, and consent form were reviewed and approved by our institutional review board.

\section{Results}

Although originally intended to be a 1-year demonstration program, the project has been funded for a second year. The results presented here are for the first 12 months of the project.

\section{Sample Description}

A total of 36 patients were enrolled by the end of the first 12 months and had attended at least one DIGMA. The median total cost of ED and inpatient services provided to this cohort during the 12 months before enrollment was \$1167. Compared with the larger cohort from which this group was recruited, the intervention group was significantly older and tended to use hospital services more intensively (see Table 1).

All but one of the 36 patients had significant poorly controlled medical or behavioral health issues at the time of enrollment. Twenty-eight of the 36 patients $(78 \%)$ had both significantly uncontrolled physical and behavioral health problems; the types and frequency of problems are listed in Table 2. The median number of chronic medications was 5 (range, $0-16$ ).

Only 4 of the participants were employed in any capacity at enrollment; 7 were homeless or living in a shelter. Fourteen reported having a PCP as a source of care, which was confirmed by obtaining medical records; an additional 3 patients had just been discharged from a primary care practice for inappropriate behavior just before enrollment. The remaining patients did not identify any PCP they had seen in a least the previous year.

Table 1. Comparison of Patients Enrolled in Treatment Group to Total Cohort

\begin{tabular}{|c|c|c|c|c|c|}
\hline \multirow[b]{2}{*}{ Variable } & \multicolumn{2}{|c|}{ Treatment Group } & \multicolumn{2}{|c|}{ Total Cohort } & \multirow[b]{2}{*}{$P^{*}$} \\
\hline & $\mathrm{n}$ (Median) & IQR & $\mathrm{n}$ (Median) & IQR & \\
\hline Age & $34(45.0)$ & 12 & $255(32.0)$ & 17 & $<.001$ \\
\hline Previsits per month & $34(0.58)$ & 0.17 & $254(0.58)$ & 0.33 & .810 \\
\hline Precharges per month & $34(1167)$ & 1133 & $255(628)$ & 718 & .002 \\
\hline Sex & $\mathrm{n}(\%)$ & & $\mathrm{n}(\%)$ & & $P^{\dagger}$ \\
\hline Female & $15(44.4)$ & & $130(51.0)$ & & .449 \\
\hline Male & $19(55.6)$ & & $125(49.0)$ & & \\
\hline
\end{tabular}

${ }^{*}$ Rank sum.

${ }^{\dagger}$ Two proportion z test.

$\mathrm{IQR}$, interquartile range. 
Table 2. Enrolled Patient Problems and Frequencies

\begin{tabular}{|c|c|}
\hline Problem & Patients (n) \\
\hline \multicolumn{2}{|l|}{ Behavioral health } \\
\hline Substance abuse & 17 \\
\hline Ethyl alcohol & 7 \\
\hline Opioids & 6 \\
\hline Benzodiazepine & 1 \\
\hline Cocaine & 2 \\
\hline Methamphetamine & 1 \\
\hline Depression & 13 \\
\hline Bipolar & 10 \\
\hline GAD & 6 \\
\hline Panic disorder & 6 \\
\hline PTSD & 6 \\
\hline Psychosis & 4 \\
\hline \multicolumn{2}{|l|}{ Physical health } \\
\hline \multicolumn{2}{|l|}{ Serious/unstable } \\
\hline Chronic pain & 27 \\
\hline COPD/asthma & 6 \\
\hline Cervical/lumbar radiculopathy & 4 \\
\hline Cyclic/recurrent vomiting & 3 \\
\hline Weight loss & 3 \\
\hline Cerebrovascular accident & 2 \\
\hline Seizure disorder & 2 \\
\hline Fracture & 2 \\
\hline Sarcoidosis & 1 \\
\hline Chronic pancreatitis & 1 \\
\hline Congestive heart failure & 1 \\
\hline Malignant hypertension & 1 \\
\hline PSVT & 1 \\
\hline Hyperthyroidism & 1 \\
\hline Rheumatoid Arthritis & 1 \\
\hline \multicolumn{2}{|l|}{ Chronic/Stable } \\
\hline Tobacco abuse & 13 \\
\hline Hypertension & 7 \\
\hline Morbid obesity & 6 \\
\hline Hepatitis & 4 \\
\hline GERD & 4 \\
\hline Fibromyalgia & 3 \\
\hline Hyperlipidemia & 3 \\
\hline DJD & 2 \\
\hline Urinary incontinence & 2 \\
\hline Gout & 2 \\
\hline Hypothyroidism & 2 \\
\hline Diabetes & 1 \\
\hline Obstructive sleep apnea & 1 \\
\hline
\end{tabular}

COPD, chronic obstructive pulmonary disease; DJD, degenerative joint disease; GAD, generalized anxiety disorder; GERD, gastroesophageal reflux disease; PSVT, paroxysmal supraventricular tachycardia; PTSD, posttraumatic stress disorder.

\section{Implementation Description}

From July 1, 2010, until August 30, 2011, the team provided a total of 897 DIGMA face-toface encounters and another 854 case manager phone calls, for a total of 1751 direct patient contacts. The average number of patients attending each DIGMA averaged approximately 8 (range, 5-14. The median number of visits per month per patient was 2 (range, $0.1-14$ ), and the median number of patient contacts per month was 3.5 (range, 0.5-25).

\section{Impact}

The cohort averaged $0.58 \mathrm{ED}$ visits per person per month for the 12 months before enrollment. After enrollment, per person per month ED utilization dropped to $0.23(P<.001$; see Figure 1 and Table 3$)$. Total ED and inpatient mean charges per person per month fell from $\$ 1167$ for the 12 months before enrollment to $\$ 230$ since enrollment $(P<$ $.001)$.

Before enrollment only 4 patients reported any current gainful employment. Since enrollment, 14 report at least part-time work. Seven patients were homeless or living in a shelter at the time of enrollment. As of August 30, 2011, only 1 patient was without stable housing.

The total annualized direct costs of the program, including the value of the donated physician time, was $\$ 66,000$. This excludes the in-kind contributions of the Free Clinic and the hospital, which are not tracked and cannot be easily estimated.

\section{Discussion}

Bridges to Health is a unique primary care team designed to serve a cohort of patients with complex medical and behavioral health problems and limited personal and social resources to manage these conditions. The addition of the DIGMA to more traditional case management seems to be a robust model of care for this population of patients.

The DIGMA is ideally suited to build effective relationships with patients. We did not put any limits on the number of DIGMAs patients could attend; our experience was that patients made frequent use of the group early on as the team was addressing their problems, with attendance becoming less frequent as their condition stabilized. These frequent, longer visits allowed patients to develop more meaningful relationships quickly with the care team and other patients, 
Figure 1. Hospital use (emergency department [ED] and inpatient [IP]).

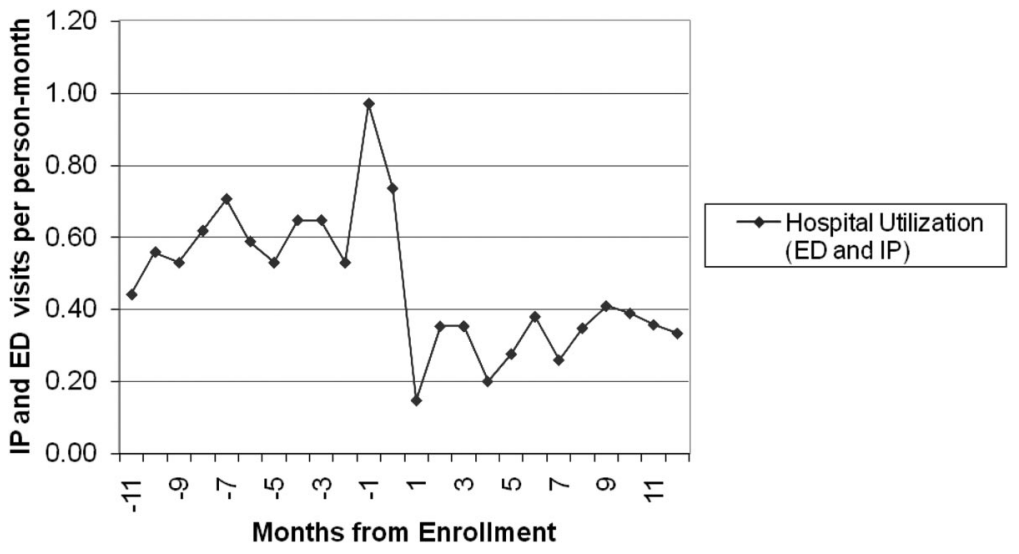

and they allowed the care team to gain a fuller understanding of the myriad issues confronting each client, which lead to opportunities to address these issues more quickly.

As we enrolled patients into the group we found that, despite a broad range of medical and behavioral health problems, the common feature they shared and what ultimately served to bring them together as a group was their status of being "on the fringe," as they described themselves. Nearly every patient had experienced a number of barriers and frustrations in accessing medical care that the DIGMA team seems to have successfully addressed. More than half the patients identified a PCP they had accessed at least once during the previous year; nevertheless, as a group these patients used ED services more than 23 times a month during the year before enrollment. The emotional support provided by the group seemed to be a key factor in assisting patients to begin to find solutions to their complicated health and social problems.

The frequency at which patients seemed to desire contact with the care team, particularly new enrollees who tended to be in crisis and unstable, would be difficult to accommodate in a typical individual appointment or fee-for-service model. We were able to provide 705 face-to-face visits with 3 care providers in only 108 one-hour sessions over 12 months, which proved to be an efficient way to provide care.

We observed that a substantial number of the group found at least part-time employment after their conditions were stabilized, and all but one was able to secure stable housing. We attribute these gains to assisting patients to access other community services and a generally positive and hopeful culture that developed within the group.

Table 3. Comparison of Treatment Group (Before vs After) and Treatment With Cohort Subset

\begin{tabular}{|c|c|c|c|c|c|c|c|}
\hline \multirow[b]{2}{*}{ Variable } & \multicolumn{2}{|c|}{ Treatment } & \multirow[b]{2}{*}{$P^{*}$} & \multicolumn{2}{|c|}{ Control } & \multirow[b]{2}{*}{$P^{*}$} & \multirow{2}{*}{$\frac{\text { Comparison }}{P^{\dagger}}$} \\
\hline & n (Median) & IQR & & n (Median) & IQR & & \\
\hline Age, years & $34(45.0)$ & 12 & & $36(33.0)$ & 17.0 & & $<.001$ \\
\hline \multicolumn{8}{|c|}{ Visits per month } \\
\hline Before & $34(0.58)$ & 0.33 & & $36(0.58)$ & 0.21 & & .891 \\
\hline After & $34(0.23)$ & 0.50 & & $36(0.42)$ & 0.50 & & .007 \\
\hline Difference & $34(0.35)$ & 0.33 & $<.001$ & $36(0.13)$ & 0.50 & .084 & .005 \\
\hline \multicolumn{8}{|c|}{ Charges per month (CA\$) } \\
\hline Before & $34(1167)$ & 1132 & & & & & \\
\hline After & $33(230)$ & 899 & & & & & \\
\hline Difference & $33(637)$ & 1104 & $<.001$ & & & & \\
\hline
\end{tabular}

*Signed rank.

${ }^{\dagger}$ Rank sum.

IQR, interquartile range. 
The DIGMA for this population is quite different from most other group medical visits or group therapy visits. Group medical visits that involve patients who share a single chronic illness, such as diabetes or heart failure, allow a team to prepare educational materials and structure. Similarly, scheduled group medical visits give the team control of who is invited to the group and how many are likely to attend any particular group. The DIGMA can be highly variable both in the number of attendees and the range of issues that need to be addressed during the visit. Our team managed these challenges by attempting to maintain a familiar structure to each group appointment to encourage a group "norm" to develop; patients quickly learn what to expect from the group experience and adapt. Although we may not know exactly who will show up for a particular visit, we can get an idea of at least some of the members that day by seeing who is in the waiting room before the visit or who has contacted the care manager between visits. A short huddle before the visit helps bring the team up to date about particular patient interactions or events that may have occurred since the last visit and to formulate a general approach and plan for the group that day.

Although the group visit is not group therapy, there are obvious parallels because the model seems to use the power of group norms, support, and feedback to help patients help themselves. Time permitting, we attempt to do at least one short group exercise during every visit to improve relaxation, control of emotions, more positive thoughts, and skills for dealing with difficult relationships and problem solving. Group cohesion grows out of the common experience of isolation that frequently accompanies chronic illness and poverty and the shared social experiences of group members, which gradually can evolve into a healing culture with behavioral health group facilitation. There is limited time for therapy per se, but it does occur individually when the need arises.

Our experience with this group of patients is that their needs are diverse and complicated and that their paths to recovery can be equally complicated. Care management is a vital piece of the puzzle, pulling together community resources without which recovery would be impossible. The care manager is an experienced, calm, trusted professional patients can call when they are frightened or in crisis between groups visits, which is often the difference between going to the ED to seek imme- diate care or waiting a day or 2 until the next group visit. Successful case management also includes assisting with teaching some of these patients basic life skills, for example, not to find housing for them, but rather direct them where to go to get housing assistance. These small, positive steps are then shared with the group, which further reinforces a growing sense of confidence.

\section{Limitations}

Although we attempted to contact every patient in the larger group of 255 patients, we were only able to reach 147 and enrolled only 36. Although the patients we enrolled were older and had significantly more costly hospital care than the larger group as a whole, they were probably more motivated to change behavior given their willingness to participate in the project. It is likely that most hospitals have similar groups of patients, of which a portion would be good candidates for a program such as this, but we cannot conclude that similar savings could have been achieved if we had been able to reach the entire cohort.

The program has been in operation for only 12 months, and not all our participants have completed a full year. We do not yet know if these outcomes will be sustained over time or if the patients will relapse into previous behaviors if and when they are graduated back into usual care models.

Although there is good evidence that the care management component of our model can be replicated in a number of other settings, the DIGMA model of care for this population has not yet been widely tested. Also, we cannot know for sure that patients did not use other EDs in the region because we do not have access to those records. Based on previous hospital use patterns, the difficulties most of these clients had with transportation, and the fact that clients would tell us freely if they had gone to the ED, we believe this happened rarely if at all. Although some insured patients with high utilization of ED and inpatient use may reduce this pattern of utilization through other means, such as obtaining insurance, securing a source of primary care, or recovery from a relapsing illness, data from our control group suggests this effect is modest.

\section{Conclusions}

Coordinated, team-based DIGMAs integrating medical and behavioral health care and care man- 
agement services seem to be a cost-effective model to reduce ED visits and combined ED and inpatient hospital charges among some patients with complex behavioral health and medical needs, even those who report access to traditional primary care. The model facilitates closer team member communication and can engage patients in supporting their own and others' recovery efforts. Professionals wishing to work with a cohort of patients with complex needs must be experienced, flexible, and prepared to deal with a number of unexpected events during the group appointment.

The authors thank Caitlin Crane and Katherine Sloss, MD, for extensive assistance in the editing of the manuscript.

\section{References}

1. Skinner J, Carter L, Haxton C. Case management of patients who frequently present to a Scottish emergency department. Emerg Med J 2009;26:103-5.

2. Wetta-Hall R. Impact of a collaborative community case management program on a low-income uninsured population in Sedgwick County, KS. Appl Nurs Res 2007;20:188-94.

3. Bird SR, Kurowski W, Dickman GK, Kronborg I. Integrated care facilitation for older patients with complex health care needs reduces hospital demand. Aust Health Rev 2007;31:451-61, discussion 449-50.

4. Horwitz SM, Busch SH, Balestracci KM, Ellingson $\mathrm{KD}$, Rawlings J. Intensive intervention improves pri- mary care follow-up for uninsured emergency department patients. Acad Emerg Med 2005;12:647-52.

5. Witbeck G, Hornfeld S, Dalack GW. Emergency room outreach to chronically addicted individuals. A pilot study. J Subst Abuse Treat 2000;19:39-43.

6. Shah R, Chen C, O'Rourke S, Lee M, Mohanty SA, Abraham J. Evaluation of care management for the uninsured. Med Care 2011;49:166-71.

7. Sadowski LS, Kee RA, VanderWeele TJ, Buchanan D. Effect of a housing and case management program on emergency department visits and hospitalizations among chronically ill homeless adults: a randomized trial. JAMA 2009;301:1771-8.

8. Shumway M, Boccellari A, O'Brien K, Okin RL. Cost-effectiveness of clinical case management for ED frequent users: results of a randomized trial. Am J Emerg Med 2008;26:155-64.

9. Okin RL, Boccellari A, Azocar F, et al. The effects of clinical case management on hospital service use among ED frequent users. Am J Emerg Med 2000; 18:603-8.

10. Pope D, Fernandes CM, Bouthillette F, Etherington J. Frequent users of the emergency department: a program to improve care and reduce visits. CMAJ 2000;162:1017-20.

11. Noffsinger EB. Running group visits in your practice. New York: Springer; 2009.

12. U.S. Census Bureau. State and county quick facts. Henderson County, North Carolina. December 23, 2011. Available at http://quickfacts.census.gov/qfd/ states/37/37089.html. Accessed January 23, 2012.

13. Pardee Hospital. Pardee 2010 annual report. Available at http://www.pardeehospital.org/files/Annual_Report_ 2010_web.pdf. October 2011. 